\title{
Polyphase Multipath Radio Circuits for Dynamic Spectrum Access
}

\author{
Eric A. M. Klumperink, Rameswor Shrestha, Eisse Mensink, Vincent J. Arkesteijn, and Bram Nauta, \\ University of Twente
}

\begin{abstract}
Dynamic access of unused spectrum via a cognitive radio asks for flexible radio circuits that can work at an arbitrary radio frequency. This article reviews techniques to realize radios without resorting to frequency selective dedicated filters. In particular, a recently proposed polyphase multipath technique canceling harmonics and sidebands is discussed. Using this technique, a wideband and flexible power upconverter with a clean output spectrum has been realized on a CMOS chip, aiming at flexible radio transmitter application. Prototype chips can transmit at an arbitrary frequency between DC and $2.4 \mathrm{GHz}$. Unwanted harmonics and sidebands are more than $40 \mathrm{~dB}$ lower than the desired signal up to the 17th harmonic of the transmit frequency.
\end{abstract}

\section{INTRODUCTION}

Cognitive radios aim at exploiting the scarce radio spectrum in a smart flexible way. Traditional TV bands between 50 and $900 \mathrm{MHz}$ are currently being freed for new applications. New licensed users are planned (e.g., digital video broadcast - handheld, DVB-H), but, in addition, new ideas for more flexible use of the spectrum are explored [1]. For higher frequencies similar ideas are developed. Standardization activities for IEEE 801.22 and $801.16 \mathrm{~h}$ are already underway. In general, regulatory organizations seem to be moving in the direction of providing more freedom to new standards, where only a minimum set of requirements are enforced. For example, regulations might allow white spectrum to be exploited, where "detect and avoid" rules are defined (e.g., response times, maximum interference levels to incumbent services). This will lead to new radio systems with different requirements on the radio software and hardware. In this article we mainly focus on the impact of cognitive radio system requirements on the physical layer (PHY), and especially the radio frequency (RF) hardware. At the end of the article we discuss some issues relating to higher layers of cognitive radio sys- tems. For now, the focus will be on radio hardware aiming at highly flexible dynamic spectrum access.

To allow for flexible spectrum access, a flexible radio hardware platform is desired, allowing for flexible choice of the radio frequency depending on free available spectrum. Traditional radio hardware is primarily optimized for cost and low power, but not for flexibility. Low power is often achieved using inductors and capacitors in resonating circuits with a high quality factor, dissipating only a fraction of the maximum energy stored in the reactive components. However, such circuits only work effectively in a narrow band around their resonance frequency, and are hence application specific for a certain band. Micro-electro-mechanical system (MEMS) technology may help to relax this problem; however, for reasons of cost and form factor, fully integrated solutions in mainstream complementary metal oxide semiconductor (CMOS) technology are preferred. Thus, we focus in this article on CMOS circuits and integrated circuit (IC) architectures. We analyze the desired functionality of the radio interface for dynamic spectrum access, and look at some feasibility bottlenecks induced by CMOS circuit properties, like timing jitter, nonlinearity, and time variance. Some possible solution directions are reviewed, including a recently proposed polyphase multipath technique. This technique enables the realization of a highly flexible radio transmitter for the DC-2.4 $\mathrm{GHz}$ range on a CMOS chip without dedicated filters. We discuss some possible application directions of this transmitter in cognitive radio systems at the end of this article.

\section{FLEXIBLE RX/RFS: MORE THAN AN ADC}

Figure 1 shows a high level functional block schematic of a cognitive radio. It consists of an antenna connected to a radio receiver (RX), a radio transmitter (TX), and an RF scanner (RFS). A baseband processing and control unit processes the spectral information, and decides which frequency is free for use. It controls the 
frequency synthesizer to generate the desired $\mathrm{RF}$ carrier, sends bits to the TX, and receives bits from the RX.

Ideally, a cognitive radio should be free to communicate wherever free spectrum is available (i.e., be very flexible in terms of the transmit frequency). This suggests a wideband radio receiver should be used for detecting free spectrum and receiving data, in contrast to traditional narrowband radio systems. On a high abstraction level, a cognitive radio can then be considered as an analog-to-digital converter (ADC) for the RX and RFS blocks, and a ditital-to-analog converter (DAC) for the TX block. All radio signal processing can then be done in the digital domain, with maximum flexibility.

To judge the feasibility of a wideband ADCbased receiver, Walden's overview article on ADCs is useful [2]. Consider, for instance, a radio communication receiver operating at popular radio frequencies between 0.05 and 6 GHz. Typical transmit power levels for current radio standards in that range are in the range of $10 \mathrm{~mW}$ up to more than $1 \mathrm{~W}$. The radio path loss strongly varies from case to case, but it is quite common to receive radio antenna voltages in the range from $1 \mu \mathrm{V}$ up to $100 \mathrm{mV}$. To detect a weak $1 \mu \mathrm{V}$ signal in the presence of a $100 \mathrm{mV}$ interferer, we need an ADC with more than $100 \mathrm{mV} / 1 \mu \mathrm{V}=100.000$ detection levels, roughly $2^{16}$ levels (16 bits). To observe 5 $\mathrm{GHz}$ signals, the ADC should at least take 10 Gsamples/s. Assuming for a moment this is technically feasible, at a (rather optimistic) energy of $1 \mathrm{pJ}$ per conversion [2], this leads to a power consumption of $10^{10}$ samples/s $\times 2^{16}$ levels $\times 10^{-12} \mathrm{~J} \approx 1 \mathrm{~kW}$ ! The energy per conversion decreases only slowly over time because analog accuracy requirements are involved, which do not benefit much from Moore's law. Note also that the actual radio bandwidth of interest is typically orders of magnitude lower than the radio carrier frequency. This makes "full-Nyquist" A/D conversion really overkill and a waste of power, even if it became technically feasible. Thus, we feel there is a need for architecture innovations to make highly flexible cognitive radio systems feasible.

A more realistic and still reasonably flexible approach is to downconvert an RF signal of interest to DC ("zero-IF architecture"), reduce its bandwidth and dynamic range by low-pass filtering, and then do the $\mathrm{A} / \mathrm{D}$ conversion at a rate and resolution that are feasible at 10-100 mW ADC power. Recently a software defined front-end using this approach for the 500 $\mathrm{MHz}-5 \mathrm{GHz}$ band has been proposed [3]. It uses a wideband low noise amplifier exploiting thermal noise cancellation [4], followed by a downconversion mixer. However, as there is hardly any RF prefiltering, the linearity requirements on the RF front-end are very high. Moreover, wideband downconverters using hard-switched mixers are plagued by spurious responses (i.e., they downconvert not only the wanted RF-band, but also its harmonics). Thus, harmonic rejection mixers are needed, as proposed in $[3,5]$. We address this harmonic rejection mixing later in this article when dealing with upconversion mixers.

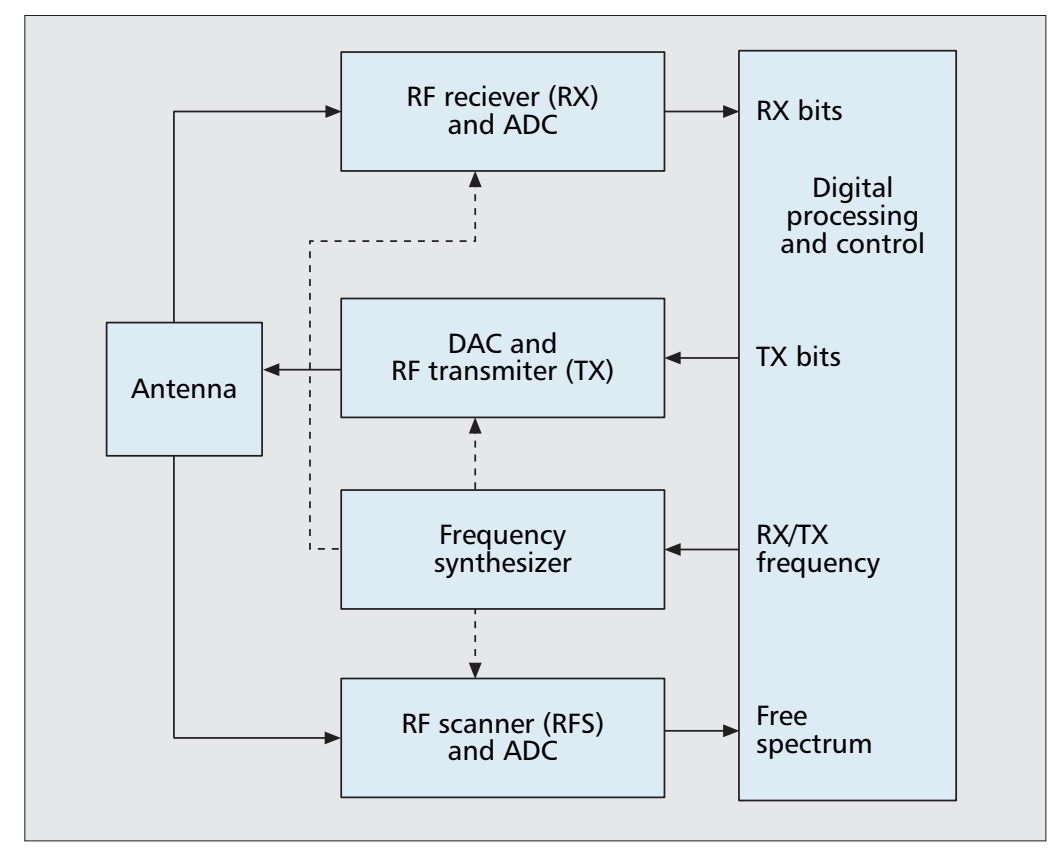

Figure 1. Block diagram of a cognitive radio system for dynamic spectrum access.

\section{SAMPLING CLOCK JITTER REQUIREMENTS}

Instead of a mixer, a sampler can also be used for frequency downconversion. Whereas full Nyquist rate A/D conversion of gigahertz signals is currently far from feasible, sampling at $\mathrm{GHz}$ rates without high resolution quantization is practical, as demonstrated for a Bluetooth and GSM receiver [6]. These receivers sample the antenna signal at RF and then process it in the charge domain via passive switched capacitor circuits. Via decimation with internal anti-alias filtering, the sample rate is reduced to a sufficiently low rate to do A/D conversion at acceptable power consumption [6].

The sampling at RF might surprise people who work on low jitter sampling clocks for highspeed ADCs, where clock jitter requirements can be a feasibility bottleneck. This is because timing uncertainty shifts the sampling moments, introducing significant amplitude errors especially for high-amplitude high-frequency signals. To keep these errors from degrading the resolution of the ADC, an extremely low root mean square RMS jitter of less than 11 fs would be needed for an 11-bit ADC sampling a $6 \mathrm{GHz}$ full swing sine wave signal [7].

Fortunately, for radio receiver applications, sampling jitter turns out to be much less harmful. This is because radio signals are narrowband in nature, so only the noise level in the wanted channel band is relevant. Jitter in a sampling clock introduces noise at the output of the sampler which strongly varies with frequency and is mainly concentrated around strong high-frequency interferers [7]. The roll-off with frequency distance from the interferer depends on the shape of the phase noise spectrum of the sampling clock. Overall, the requirement on the sampling clock jitter is close to what is needed for traditional mixer-based receiver systems lim- 


As digital circuits
benefit from
Moore's law,
we strongly prefer
flexible digital
synthesizer
techniques over
analog sine wave
generation.
However, this means
we have to find a
solution to suppress
unwanted
harmonics.

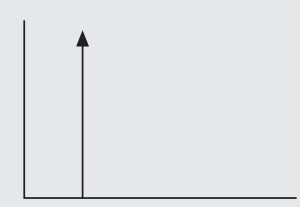

$\omega$
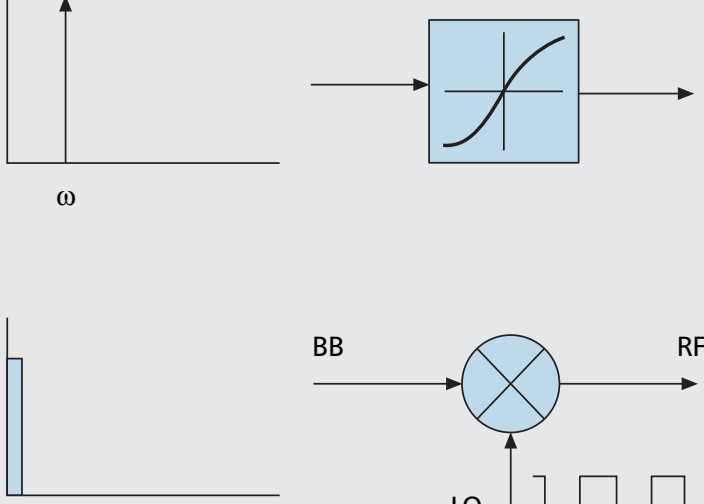

Base Band (BB)

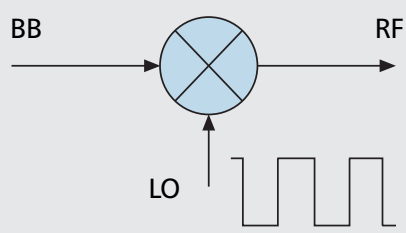

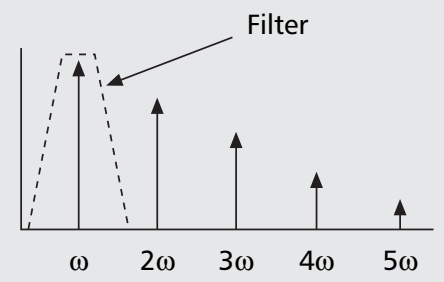

Digital synthesizer

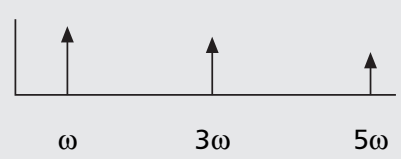

Figure 2. Nonlinearity and time variance due to switched mixers generate unwanted spectral components, which are traditionally removed by dedicated band-pass filters.

ited by reciprocal mixing [7]. Calculation for a Bluetooth receiver shows that 1.3 ps RMS-jitter can be accepted, which is more than two orders of magnitude easier than corresponding $A D C$ clock jitter specs [7]. Thus, jitter is not as big a problem as often thought, opening the door for radio architectures exploiting high-speed sampling like in [6].

\section{FLEXIBLE TX: MORE THAN A DAC}

Realizing a flexible transmitter using a DAC seems possible in principle, as the dynamic range of a transmitted signal is typically significantly lower than the dynamic range of a received signal. However, apart from the useful TX signal, many other spurious components may be produced. As a radio transmitter should produce significant output power, typically in the range between $1 \mathrm{~mW}$ and $10 \mathrm{~W}$, power drivers and power amplifier circuits with transistors working at large signal swings are used. Thus, nonlinearity of the transistors plays an important role, resulting in harmonics (Fig. 2) and intermodulation distortion products at many unwanted frequencies [8]. As the power efficiency of most amplifiers increases for higher signal swings, it is desirable to drive the amplifiers to a level close to their compression point. However, in practice significant backoff is needed [9] to suppress distortion products sufficiently at the cost of efficiency.

Apart from nonlinearity, a time-variant transfer function can also introduce many unwanted frequency components. Ideal DACs and hardswitched mixers can be modeled as linear timevariant circuits, with a linear transfer from input to output that changes instantaneously with the state of the clock signal. For simplicity, we only discuss the case of an upconversion TX mixer here, but similar conclusions hold for a DAC. The mixer is shown in Fig. 2, where an ideal 50 percent square wave switching between +1 and -1 models the hard-switching mixer operation. This square waveform has odd harmonics with a relative strength of $1 / 3,1 / 5,1 / 7, \ldots$ compared to the fundamental. Thus, the 9 th harmonic is still stronger than $-20 \mathrm{~dB}$ compared to the fundamental.

In order to avoid harmonic mixing, the input signal could be multiplied by a sine wave signal using a highly linear multiplier. However, realizing a linear multiplier is much more difficult than a hard-switched mixer, and the generation of a clean sine wave is problematic, especially when a large frequency range is involved. Typical sine wave oscillators (e.g., LC oscillators) have only a limited tuning range on the order of 5-50 percent. If a larger tuning range is needed, digital dividers are commonly used to divide the voltage controlled oscillater (VCO) frequency to an appropriate value. As digital circuits benefit from Moore's law, we strongly prefer flexible digital synthesizer techniques over analog sine wave generation. However, this means we have to find a solution to suppress unwanted harmonics.

In traditional radio transmitters, these unwanted products are rejected using dedicated band-pass filters typically implemented using inductors and capacitors (LC filters). We like to avoid such filters on CMOS chips, as they require high-quality inductors that are difficult to implement and/or take large chip area. For dynamic spectrum access, such filters are even more problematic as LC band-pass filters work at a fixed frequency related to the LC resonance frequency, which limits flexibility in choosing a TX frequency. The next section discusses a recently proposed polyphase multipath tech- 

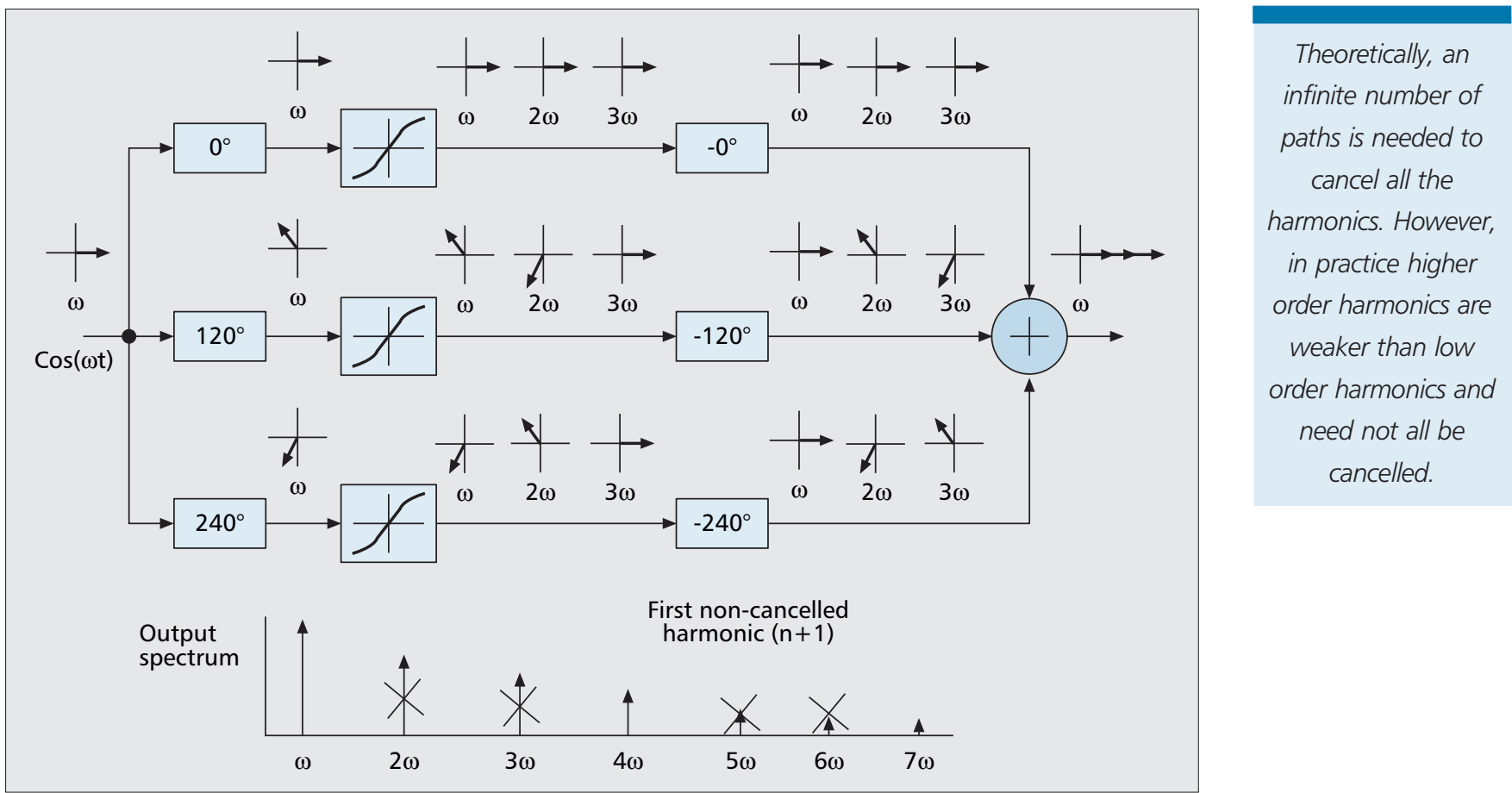

Figure 3. Polyphase three-path circuit with harmonic cancellation except for harmonics $\mathrm{i} \times \mathrm{n}+1$ (in this case $\mathrm{n}=3$, so harmonics $1,4,7, \ldots$ are not cancelled).

nique to eliminate these filters or relax their requirements significantly.

\section{Polyphase Multipath Circuits FOR SPECTRAL PURITY ENHANCEMENT}

Figure 2 shows a nonlinear circuit excited by a single sine wave at $\omega$, producing a wanted output signal at $\omega$ but also unwanted harmonic distortion at $2 \omega, 3 \omega, 4 \omega, \ldots$. Figure 3 shows a polyphase three-path circuit, canceling many harmonics of $\omega$ [10]. The basic idea is to divide a nonlinear circuit of Fig. 2 into $n$ equal smaller pieces, and apply an equal but opposite phase shift before and after each nonlinear circuit. If the phase shift in path $i$ is $(i-1) \times \varphi$, where $\varphi$ is a phase shift constant satisfying $n \times \varphi=360^{\circ}$, the circuit will produce the same wanted harmonic as Fig. 2, but cancel many higher harmonics. Mathematically this can easily be shown using a power series expansion, assuming a memoryless weakly nonlinear system. If the signal $x(t)=A \cos (\omega t)$ is applied to the input, the output of the nonlinear circuit of the ith path can be written as

$$
\begin{aligned}
& p_{i}(t)=a_{0}+a_{1} \cos (\omega t+(i-1) \varphi) \\
& +a_{2} \cos (2 \omega t+2(i-1) \varphi) \\
& +a_{3} \cos (3 \omega t+3(i-1) \varphi)+\ldots
\end{aligned}
$$

where $a_{0}, a_{1}, a_{2}, a_{3} \ldots$ are Taylor series constants characterizing the nonlinearity [8]. From Eq. 1, it can be seen that the phase of the $k$ th harmonic at the output of the nonlinear circuit rotates by $k$ times the input phase $(i-1) \varphi$. The phase shifters, $-(i-1) \varphi$, after the nonlinear blocks are required to align the fundamental components at $\omega$ in phase again.

The signals at the output of these phase shifters can be written as

$$
\begin{aligned}
& y_{i}(t)=a_{0}+a_{1} \cos (\omega t) \\
& +a_{2} \cos (2 \omega t+(i-1) \varphi) \\
& +a_{3} \cos (3 \omega t+2(i-1) \varphi)+\ldots
\end{aligned}
$$

In Eq. 2 the phase of the fundamental component is identical for all the paths, but the phases of the harmonics are different for each path. If the phase $\varphi$ is chosen such that $\varphi=$ $360^{\circ} / n$, all the higher harmonics are cancelled [10], except for the $k$ th harmonics for which $k$ equals $j \times n+1(j=0,1,2,3, \ldots)$.

The simplest example of a polyphase multipath circuit is a well-known differential circuit driven with balanced (anti-phase) input signals. It cancels all even harmonics (no cancellation of $k=j \times 2+1$, i.e., odd harmonics).

A system with three paths is shown in Fig. 3. In this case phase shifts of $0^{\circ}, 120^{\circ}$, and $240^{\circ}$ are added before the nonlinear block to paths 1,2 , and 3 respectively, and equal but opposite phases $-0^{\circ},-120^{\circ}$ and $-240^{\circ}$ behind the block. Due to the nonlinearity, the phase rotation for the kth harmonic is $\mathrm{k}$ times the input phase. Thus the respective phases at the output of the nonlinear block for path $[1,2,3]$ are $\left[0^{\circ}, 120^{\circ}, 240^{\circ}\right]$ for $\omega$, $\left[0^{\circ}, 240^{\circ}, 120^{\circ}\right]$ for $2 \omega$ and $\left[0^{\circ}, 0^{\circ}, 0^{\circ}\right]$ for $3 \omega$ products. Figure 3 also shows how the phases of the harmonics at the output of each path combine. Only the fundamental components add up in phase, while the vectors for the second and third harmonics create a "balanced structure" at the output, resulting in a zero sum (cancellation). However, the fourth harmonic components will align in phase again, and will add up 
As upconversion is

desired in a

transmitter circuit anyway, this fits nicely to our goal. However, a mixer produces not only a sum frequency but also a difference frequency. Usually only one of these is the wanted signal, while the other ("the image") needs to be suppressed.

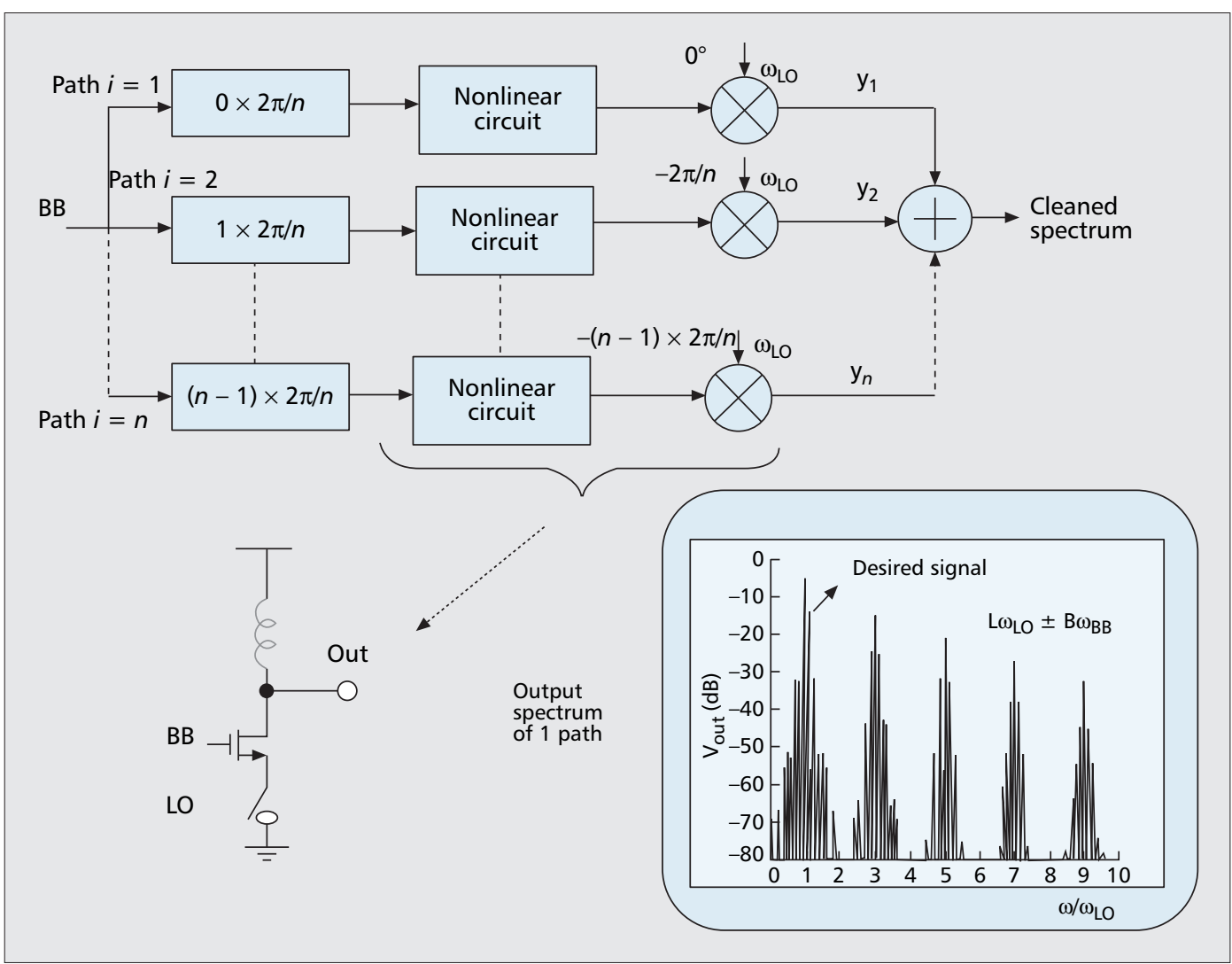

Figure 4. Polyphase n-path transmitter using mixers as phase shifters. Each path can be as simple as a transistor with switch. Due to timevariance (switching) and nonlinearity the output spectrum for 1 path has many harmonics and sidebands, which can be removed in the npath system.

like the fundamental. The output spectrum in the lower part of Fig. 3 shows that the 2nd, 3rd, 5 th, 6 th, and so on harmonics are cancelled, and the first non-cancelled harmonic is the fourth for a three-path system. Similarly, for a four-path system the first non-cancelled harmonic will be the fifth harmonic, and in general for an $n$-path system the $(n+1)$ th harmonic is the first noncancelled harmonic. Theoretically, an infinite number of paths is needed to cancel all the harmonics. However, in practice higher order harmonics are weaker than low order harmonics and need not all be cancelled. Also, in practice some filtering will always be present, for example, due to the limited bandwidth of an antenna or the speed limitations in a circuit. Moreover, mismatches will put a practical limit on what is feasible [10].

If the nonlinear system is excited by a twotone input signal $x(t)=A_{1} \cos \omega_{1} t+A_{2} \cos \omega_{2} t$, besides harmonics the output will also contain intermodulation products at new frequencies $p \omega_{1}$ $+q \omega_{2}$, where $p$ and $q$ identify harmonics of $\omega_{1}$ and $\omega_{2}$, respectively, and can be positive or negative integer numbers. It can easily be shown that many intermodulation products are cancelled, except if $p+q$ equals $j \times n+1$ (where $j$ $=0,1,2,3, \ldots)$.

\section{Mixer: Phase ANd Frequency Shifter}

To realize wideband harmonic rejection using a polyphase multipath system, we need very wideband phase shifters before and after the nonlinearity. This is because all phase shifters need to have a constant phase shift over all relevant frequencies involved in the cancellation process. In a digital signal processing (DSP)-intensive radio transmitter, DSP techniques can be exploited to realize phase shifters before $\mathrm{D} / \mathrm{A}$ conversion and nonlinear power amplification. Therefore, a good solution can be to shift this polyphase generation problem to the digital domain, and use a DSP followed by multiple DACs to generate multiphase baseband signals. However, behind the nonlinear element we are in the analog domain, and there can be many harmonics. In that case cancellation of a multitude of harmonics requires constant phase shift over many octaves of frequency.

A very wideband phase shifter can be implemented with a mixer, since a mixer as shown in Fig. 2 transfers phase information of both the baseband (BB) and local oscillator (LO) port to the output. Whatever phase is added to the LO signal will appear at the output of the mixer. So by replacing the second set of phase shifters in Fig. 3 with mixers, as shown in Fig. 4, we can achieve a wideband phase shift but simultaneously will get frequency conversion. As upconversion is desired in a transmitter circuit anyway, this fits nicely with our goal. However, a mixer produces not only a sum frequency but also a difference frequency. Usually only one of these is the wanted signal, while the other (the "image") needs to be suppressed. Moreover, the LO signal is usually a square wave containing many harmonics, because flexible frequency synthesizers rely on digital dividers, as discussed in 


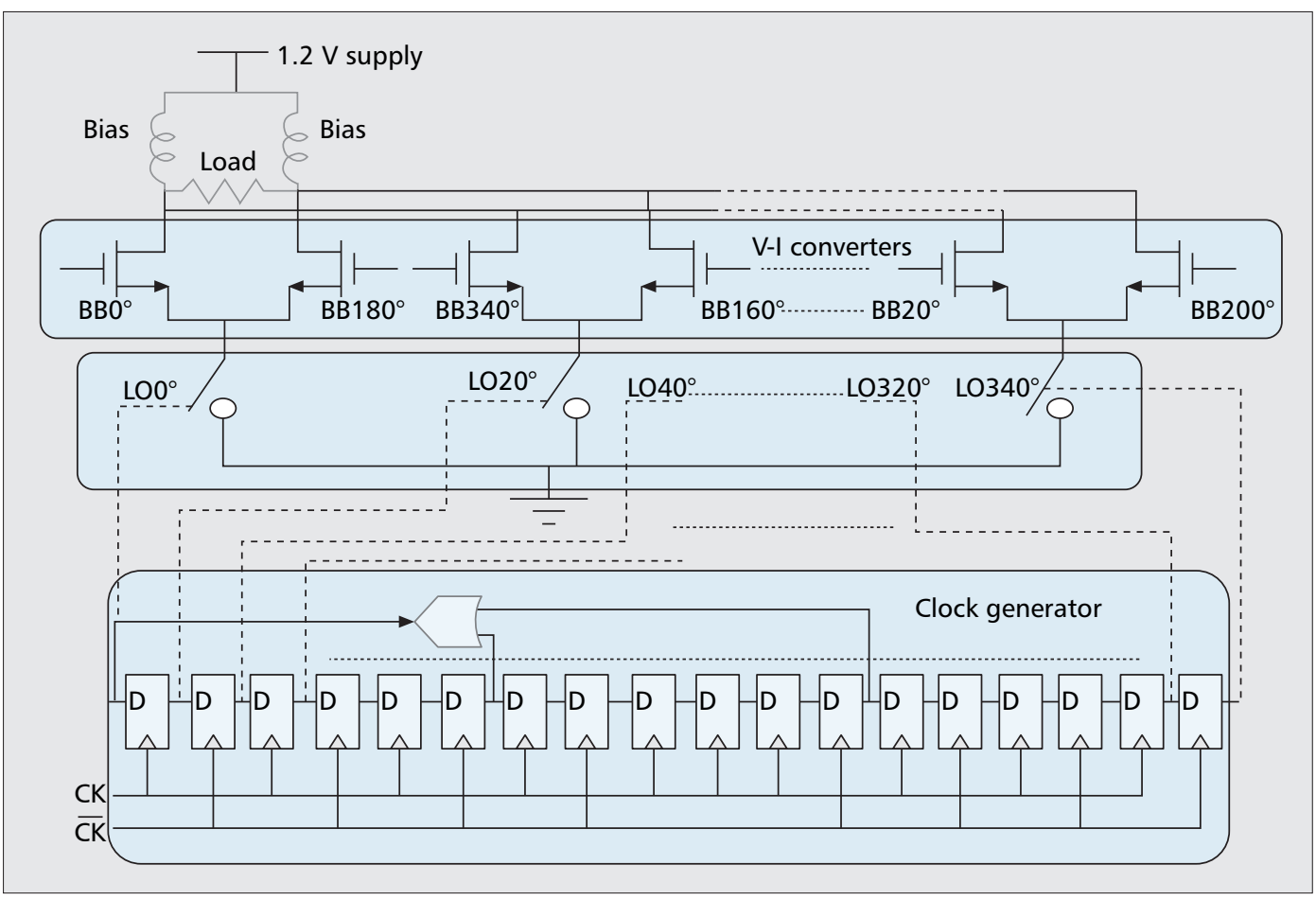

To demonstrate the

feasibility of a highly

flexible multipath

transmitter, we

designed a power

upconverter in a

$0.13 \mathrm{~mm}$ CMOS

process, covering all

frequencies up to

2.4GHz. To show

wideband spectral

cleaning we

designed an

18-path system.

Figure 5. Circuit concept of an 18-path power upconverter.

the previous section. For power efficiency it is also highly desired to use a switching mixer and a large BB signal swing (e.g., a single transistor with switch as shown in Fig. 4). Thus, the output spectrum for one path will now contain a forest of harmonics and sidebands, as shown in Fig. 4 for the case with a single-tone BB signal. Spectral components occur at frequencies $L \omega_{\mathrm{LO}} \pm$ $B \omega_{\mathrm{BB}}$, where $L$ and $B$ are integers, due to the multiplication of the square wave $\mathrm{LO}$ with the baseband input signal BB, and also the nonlinearity of the circuit. In the next section we see how we can exploit the polyphase multipath technique to cancel almost all the unwanted components.

\section{FiLTERLESS POWER UPCONVERTER}

A power upconverter combines the functionality of a power amplifier and upconversion mixer. The PA and mixer can be as simple as shown in Fig. 4, which is equivalent to first amplification and then mixing. Here the PA is a single transistor operating as a transconductor (V-I converter), which is switched on and off by the LO signal via a switch ( $N$-channel metal oxide semiconductor [NMOS] transistor driven by a digital inverter). Thus, the V-I conversion and upconversion is done in the same circuit via a switched transconductor mixer [12]. With respect to efficiency this circuit resembles a single transistor (class A) power amplifier. However, due to the polyphase multipath technique, distortion products are cancelled, and larger signal swings can be tolerated, improving efficiency.

Unfortunately, a few problematic products still remain present at the output. Since we have two input ports now (BB and $\mathrm{LO}$ ), and mixing produces several sum and difference fre- quencies, a slightly different condition for noncancelled products is found [10,11] $(L=j \times n$ $+B$, where $j=\ldots .-2,-1,0,1,2 \ldots$, and $B$ is a positive or negative integer number). Especially the $3 \omega_{\mathrm{LO}}+3 \omega_{\mathrm{BB}}$ is troublesome because the 3rd order distortion term is usually much stronger than higher order distortion components [9] and is also close to the desired signal. It cannot be cancelled with any number of paths as all products for which $L=B$ are not cancelled $(j=0$ case, so independent of $n)$. To eliminate the strong $3 \omega_{\mathrm{LO}}+3 \omega_{\mathrm{BB}}$ terms, the duty cycle of the LO was chosen to be $1 / 3$ [11]. By doing so, the 3rd, 6th, 9th, and so on harmonic terms disappear from the Fourier series expansion; however, some even order terms appear. Fortunately, it is quite easy to cancel even order products by using a differential baseband input (balancing).

\section{CMOS DEMONSTRATOR IC}

To demonstrate the feasibility of a highly flexible multipath transmitter, we designed a power upconverter in a $0.13 \mu \mathrm{m}$ CMOS process, covering all frequencies up to $2.4 \mathrm{GHz}$. To show wideband spectral cleaning we designed an 18-path system that can clean up the spectrum up to the 17 th harmonic.

Figure 5 shows the 18-path power upconverter. Each path consists of a switched transconductor mixer [12] with a baseband signal applied to a differential pair, acting as a differential transconductor (V-I converter), and an LO signal driving a grounded switch. The output currents of the V-I converters are easily added by connecting them together, and the wanted output signals from all paths add up in phase. Thus, the total area and power of the 


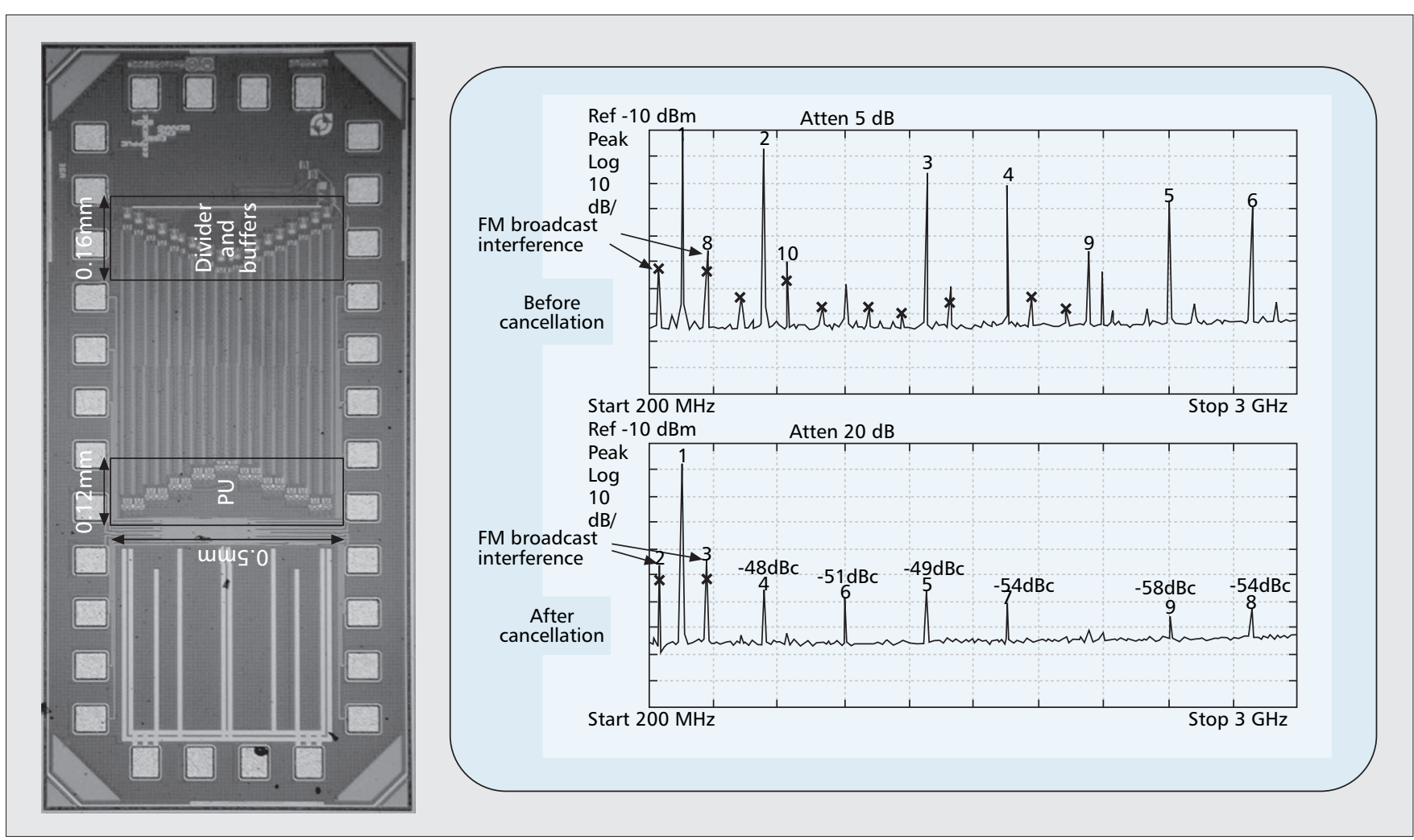

Figure 6. Photo and output spectra of the 18-path Power Upconverter (PU) chip, with out-of-band power $<-40 \mathrm{dBc}$ up to the 17th harmonic $(\mathrm{LO}=350 \mathrm{MHz})$.

power upconverter core is not increased by splitting it into 18 paths. The V-I converter transistors are biased at the supply voltage via two large inductors (Fig. 5) to increase the output swing and efficiency, as is commonly done in power amplifier design. The inductance and load resistance constitute a high-pass AC coupling, which puts a lower limit on the RF frequency, but the chip itself can work at arbitrarily low frequency. Operating each individual switched transconductor mixer at the 1 $\mathrm{dB}$ compression point, the upconverter is designed for a large output swing of about 2.5 $\mathrm{V}$ differential peak-to-peak voltage, to maximize efficiency. This is close to the maximum swing that can be achieved from a $1.2 \mathrm{~V}$ supply while keeping the output transistors in strong inversion and saturation, to maintain V-I converter functionality. For a $100 \Omega$ load, the $2.5 \mathrm{~V}$ swing corresponds to roughly $8 \mathrm{~mW}$ output power. To further increase the output power without adding an external power amplifier, a transformer could be added for broadband impedance transformation while scaling up the output current via wider transistors.

To maximize the flexibility and frequency range, we implemented the LO phase generation brute force via a current mode logic shift register running at nine times the LO frequency. Although this is far from optimal for power consumption, this enabled us to evaluate the circuit for an arbitrary LO frequency between DC and a maximum given by the speed limitation of the logic used to realize the shift register. For 18 paths we need LO signals of 18 different phases $\left(0^{\circ}, 20^{\circ}, 40^{\circ}, \ldots, 340^{\circ}\right)$ with $1 / 3$ duty cycle. Apply- ing a positive and a negative clock edge alternately to successive latches in a chain of $18 \mathrm{D}$ latches (Fig. 5), 18 different phases are produced. The feedback through the NOR gate is used to make the duty cycle $1 / 3$.

In our experimental setup, the nine differential baseband voltages with different phases are generated off-chip. More work has to be done to explore the most effective way to generate multiphase baseband signals on chip via DSP techniques and multiple DACs.

The multipath technique cleans the output spectrum from unwanted harmonics, which result from the hard-switching mixer and also from nonlinearity in the switched transconductor. Simulations and measurements show that we can drive the power upconverter close to its $1 \mathrm{~dB}$ compression point with harmonics well below $-40 \mathrm{dBc}$ and realize the high $2.5 \mathrm{~V}$ output voltage swing directly over the load (e.g., antenna). Note that the two inductors are only used for biasing, not for (dedicated) band-pass filtering.

The proposed upconverter has been fabricated in a $0.13 \mu \mathrm{m}$ CMOS process and has an active area of only $0.14 \mathrm{~mm}^{2}$ (Fig. 6). It delivers $8 \mathrm{~mW}$ output power to a $100 \Omega$ off-chip load. Figure 6 shows the output frequency spectrum for a transmit frequency of $350 \mathrm{MHz}$ for one path (no cancellation) and for the complete 18-path system (lower part of Fig. 6). Clearly all problematic products are suppressed significantly. Please note that the unfortunate FM radio spurs that are modulated with our output signal are caused by a $100 \mathrm{MHz}$ high-power FM radio broadcast transmitter on the roof of our building. Overall, 10 chips were measured with spurious emissions 
$<-40 \mathrm{dBc}$ for all harmonics up to the 17th harmonic of the LO, for an LO frequency from 30-800 MHz. For higher frequencies the chip has a 6-path mode which was measured for 30 $\mathrm{MHz}-2.4 \mathrm{GHz}$ with similar rejection up to the fifth harmonic of the LO. The rejection of products related to the fundamental of the LO, like the LO feedthrough and image component, can be a few $\mathrm{dB}$ worse, but requirements on in-band products are usually less strict than for out-ofband spurious emissions.

The (drain) efficiency of the core of the power upconverter is 11 percent, which is good compared to other power upconverters, given the low harmonics. However, we used current-mode logic circuits biased at high currents at $8 \mathrm{GHz} \mathrm{LO}$ frequency. As a result the power consumption of the digital part currently dominates $(\sim 150 \mathrm{~mW})$, but we expect this to reduce significantly in future designs, by both smarter design and downscaling of the digital power via Moore's law.

\section{RAdio System AsPeCtS}

We saw that we have a power upconverter which can work at an arbitrary frequency between DC and several gigahertz, covering the most popular radio communications bands. This part of the spectrum also contains the traditional TV bands between 50 and $900 \mathrm{MHz}$, which are freed now for new applications. In these bands new licensed "primary" users are planned, but in addition new ideas for more flexible use of the spectrum by "secondary" users are explored. In general, regulatory organizations like FCC and CEPT seem to be moving in the direction of providing more freedom to new standards, where only a minimum set of requirements are enforced, Regulations might for instance allow the exploitation of white spectrum, where detect and avoid (DAA) rules are defined by the standardization bodies like the European Telecommunications Standards Institute (ETSI) (e.g., response times, maximum interference levels to incumbent services).

White space will usually not be concentrated in one particular area of the spectrum. A more likely scenario is a spectrum with a number of medium- to narrowband white segments. A cognitive radio can select a single white segment, but is then bandwidth and capacity limited. A more powerful solution would result when the cognitive radio could simultaneously use several white segments in parallel.

On the transmit side, this would require spectral shaping where power is only transmitted at frequencies that correspond to the white segments. The frequencies between should carry as little power as possible in order to minimize the impact on the incumbent services. A good candidate for obtaining this type of TX spectrum is the use of orthogonal frequency-division multiplexing (OFDM), where "spectral notches" are created by selectively removing carriers from the multicarrier spectrum that coincide with spectrum in use by primary users [13]. However, simple removal of carriers renders only limited rejection on the order of $20 \mathrm{~dB}$ [13], as every OFDM carrier has sinc-like side lobes. By smart modulation of specific carriers, it is possible to realize active interference cancellation and increase the depth of the notch to 30-40 dB [14], provided that the RF transmitter hardware offers sufficient spectral purity. The polyphase multipath transmitter discussed in the previous section can currently suppress unwanted harmonics and sidebands with about $40 \mathrm{~dB}$, which seems good enough. In principle, calibration techniques could be used to obtain even 10-20 dB more rejection. Furthermore, as no filters are used, large transmitter bandwidths in excess of 100 $\mathrm{MHz}$ can be used, while the lack of filters also allows fast on/off switching or fast frequency hopping (frequency agilility). Thus, several techniques can be used to reduce the interference to other users and exploit temporarily unused spectrum efficiently. For instance, a combination of frequency hopping with time-division duplexing in combination with OFDM as proposed in [15] could be supported. Given the output power, the current polyphase multipath transmitter in Fig. 6 seems most suitable for ad hoc short-range radio networks.

\section{CONCLUSIONS}

In this article we review some recent research results relevant to the feasibility of fully integrated CMOS cognitive radio transceivers. We motivated why an ADC and a DAC are not sufficient to realize the radio interface. Coarse power estimates show that $\mathrm{A} / \mathrm{D}$ conversion of high dynamic range radio signals at the antenna is not realistic for gigahertz radio signals. However, RF sampling is feasible, and the sampling clock jitter requirements are not as difficult as often thought, but similar to those of traditional mixer-based RF receivers. A key fundamental problem in radio circuits is their nonlinear and/or time-variant nature. As a result, they produce not only a wanted output signal, but also many unwanted harmonics and sidebands. We present a polyphase multipath technique that addresses this problem without using any dedicated filters. Using this technique, a highly flexible power upconverter has been realized in CMOS, operating at an arbitrary transmit frequency between DC and $2.4 \mathrm{GHz}$, with unwanted harmonics and sideband lower than $-40 \mathrm{dBc}$.

\section{ACKNOWLEDGMENTS}

The authors would like to thank Fokke Hoeksema and Jaap Haartsen for their contributions during discussions on this work, and Henk de Vries and Gerard Wienk for practical assistance during design and measurements. Philips Research is acknowledged for providing the silicon.

\section{REFERENCES}

[1] M. J. Marcus, "Unlicensed Cognitive Sharing of TV Spectrum: The Controversy at the Federal Communications Commission," IEEE Commun. Mag., vol. 43, 2005, pp. 24-25.

[2] R. H. Walden, "Performance Trends for Analog to Digital Converters," IEEE Commun. Mag., vol. 37, 1999, pp. 96-101.

[3] R. Bagheri et al., "Software-Defined Radio Receiver: Dream to Reality," IEEE Commun. Mag., vol. 44, 2006, pp. 111-18.
White space will

usually not be

concentrated in one

particular area of the

spectrum. A more

likely scenario is a

spectrum with a

number of

medium- to

narrowband white

segments. 
[4] F. Bruccoleri, E. A. M. Klumperink, and B. Nauta, "Wideband CMOS low-Noise Amplifier Exploiting Therma Noise Canceling," IEEE J. Solid-State Circuits, vol. 39, 2004, pp. 275-82.

[5] J. A. Weldon et al., "A 1.75-GHz Highly Integrated NarrowBand CMOS Transmitter with Harmonic-Rejection Mixers," IEEE J. Solid-State Circuits, vol. 36, 2001, pp. 2003-15.

[6] K. Muhammad, R. B. Staszewski, and D. Leipold, "Digital RF Processing: Toward Low-Cost Reconfigurable Radios," IEEE Commun. Mag., vol. 43, 2005, pp. 105-13.

[7] V. J. Arkesteijn, E. A. M. Klumperink, and B. Nauta, "Jitter Requirements of the Sampling Clock in Software Radio Receivers," IEEE Trans. Circuits and Sys. II: Express Briefs, vol. 53, 2006, pp. 90-94.

[8] W. Sansen, "Distortion in Elementary Transistor Circuits," IEEE Trans. Circuits and Sys. II: Analog and Digital Sig. Proc., vol. 46, 1999, pp. 315-25.

[9] P. B. Kenington, "Linearized Transmitters: an Enabling Technology for Software Defined Radio," IEEE Commun. Mag., vol. 40, 2002, pp. 156-62.

[10] E. Mensink, E. A. M. Klumperink, and B. Nauta, "Distortion Cancellation by Polyphase Multipath Circuits," IEEE Trans. Circuits and Sys. I: Regular Papers, vol. 52, 2005, pp. 1785-94.

[11] R. Shrestha et al., "A Multipath Technique for Canceling Harmonics and Sidebands in a Wideband Power Upconverter," 2006, IEEE Int'l. Solid-State Circuits Conf., San Francisco, CA, Feb. 6-8, 2006, pp. 452-53.

[12] E. A. M. Klumperink et al. "A CMOS Switched Transconductor Mixer," IEEE J. Solid-State Circuits, vol. 39, 2004, pp. 1231-40.

[13] T. A. Weiss and F. K. Jondral, "Spectrum Pooling: an Innovative Strategy for the Enhancement of Spectrum Efficiency," IEEE Commun. Mag., vol. 42, 2004, pp. S8-S14.

[14] H. Yamaguchi, "Active Interference Cancellation Technique for MB-OFDM Cognitive Radio," 34th Euro. Microwave Conf., vol. 2, 2004, pp.1105-08.

[15] F. Hoeksema et al., "A Node Architecture for Disaster Relief Networking," 1st IEEE Int'l. Symp. New Frontiers in Dynamic Spectrum Access Networks, 2005, pp. 577-84.

\section{BIOGRAPHIES}

ERIC A. M. KLUMPERINK [M'98, SM'06] (E.A.M.Klumperink@ utwente.nI) received his Ph.D. degree in 1997 from the University of Twente. He is currently an associate professor at the IC Design Laboratory at the same university, and involved in the CTIT Research Institute. Since 2006 he has served as Associate Editor for IEEE Transactions on Circuits and Systems II: Express Briefs. He is a co-recipient of the ISSCC 2002 Van Vessem Outstanding Paper Award.

RAMESWOR SHRESTHA received a B.E. degree (with distinction) from Kathmandu University, Nepal, in 2000 and an M.Sc. degree (with honors) from the University of Twente, The Netherlands, in 2004, both in electrical engineering. He is currently working toward a Ph.D. degree at the University of Twente. His current research is on supply modulated RF power amplifiers.

EISSE MENSINK [S'03] received an M.Sc. degree in electrical engineering (cum laude) from the University of Twente, The Netherlands, in 2003. He is currently working toward a $\mathrm{Ph}$.D. degree at the same university on the subject of highspeed on-chip communication.

VINCENT J. ARKESTEIJN received his M.Sc. degree in electrica engineering from the University of Twente in 2000 . He is currently working toward a Ph.D. degree at the same university. His research interests include analog front-ends for software defined radio.

BRAM NAUTA [SM'03] received an M.Sc degree (cum laude) in EE and in 1991 a Ph.D. degree from the University of Twente. In 1991 he joined Philips Research, and in 1998 he returned to the University of Twente as a full professor heading the IC Design group. He is a technical program member of ISSCC, ESSCIRC, and VLSI Symposium. In 2001 he became an Associate Editor for IEEE Journal of Solid-State Circuits, and in 2007 he became its Editor. He is co-recipient of the ISSCC 2002 Van Vessem Outstanding Paper Award. 